\title{
VALIDATION OF CUTTING FORCE CHARACTERISTIC FOR COMPLEX TOOLPATH
}

\author{
Henrik Tamás Sykora*, Attila Kovács, Dániel Bachrathy
}

\author{
Budapest University of Technology and Economics, Department of Applied Mechanics, Muegyetem rkp. $5 ., 1111$ \\ Budapest, Hungary \\ * corresponding author: henrik.sykora@gmail.com
}

\begin{abstract}
In the design phase of the milling process, there is a great need for the prediction of the cutting force, the required torque and power of the spindle. These informations could be used to optimize the tool path and improve the material removal rate. In this work, we present our dexel based simulation software, its modules, calculations steps and the simulation method. Different force models were analysed to describe the specific force as a function of the local chip thickness. The models were fitted to the measured force data. Then the selected force model was validated in case of a complex tool path.
\end{abstract}

KEYWORDS: milling experiment, cutting force prediction, CWE simulation, force model fitting.

\section{INTRODUCTION}

In the design of the milling process of a product, CAM (Computer-Aided Manufacturing) softwares can be used to generate a tool path, which is appropriate for the geometric requirements. However, these algorithms do not take the dynamic effects into account. In order to make optimal usage of the machines, it is indispensable to know the connection between the milling tool and workpiece (CWE - Cutter Workpiece Engagement) accurately. However, the commercially available CAM programs usually do not provide a possibility to obtain this kind of data. Even if the CWE is known, it is also essential to use an appropriate cutting force function to predict the resultant cutting forces and moments, the resultant vibrations, the surface quality and the stability properties [1, 2].

The main goal of our work is to develop a program capable of a generic 3 axis milling simulation with a cylindrical milling tool along with the computation of forces and the moments. The experimental validation of the theoretical model is also presented.

\section{Software}

The software (see in Figure 5) is made up of loosely interconnected modules, such as the solid-modeling, CWE calculation, force calculation and the graphics module. This makes the development and the extension of the program manageable. All of the settings are stored in an user friendly input file, which enables to easily set up simulations. The software was implemented in $\mathrm{C}++$ programming language and it is crossplatform (Windows, Linux). In the following sections these modules will be presented briefly.

\subsection{Dexel Method}

The solid modelling module utilises the multi-dexel solid modelling technique [3], which is a promising discretization technique for this field, because of its many advantages, such as it is relatively easy to implement compared to other techniques [4] (e.g. the constructive solid geometry technique). It is sufficiently accurate, fast and there is potential for even more speedup by utilizing the parallell processing powers of the GPUs [5], since it comprises many separately calculable parts.

The dexel model represents the solid body by choosing a plane with a grid and then storing the intersection points of the body and the infinite straight lines projected from this grid [6] as presented in Figure 1 for planar bodies. This can be extended by using three perpendicular plaines in 3D for 3 dimensional bodies (presented in Figure 2), which leads to the so-called tridexel representation.

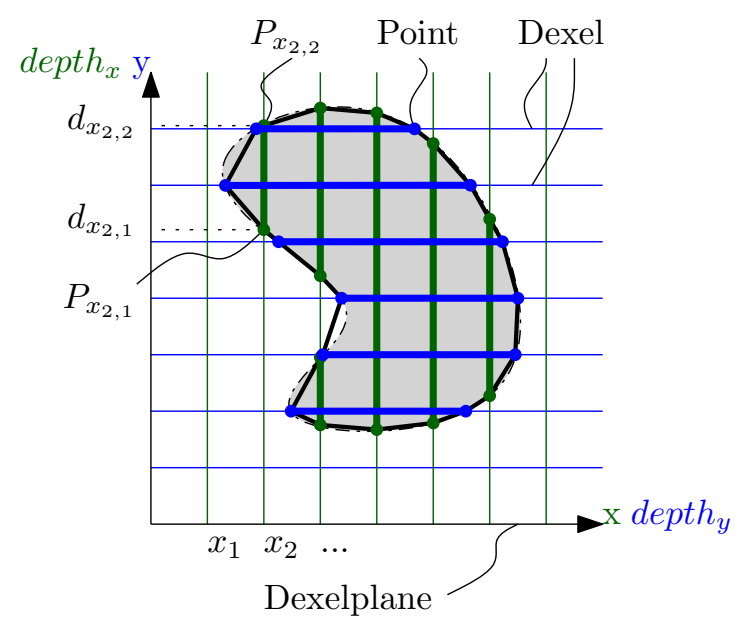

Figure 1. Multi-Dexel method in two dimensions by applying two perpendicular dexeplanes (blue and green).

The definition of boolean operations between the tridexels (subtraction, intersection, union) is also nec- 

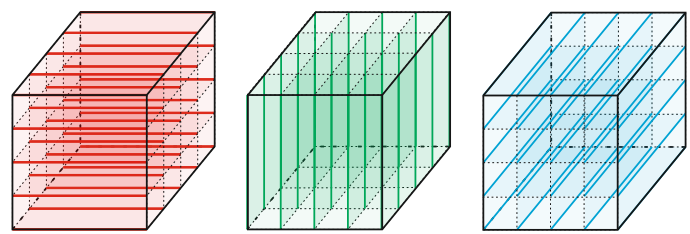

Figure 2. Three dexel planes in the Tri-Dexel method to represent spatial bodies.

essary. This is done by defining the operations between individual dexels. The concept of the operations between dexels is shown in Figure 3.

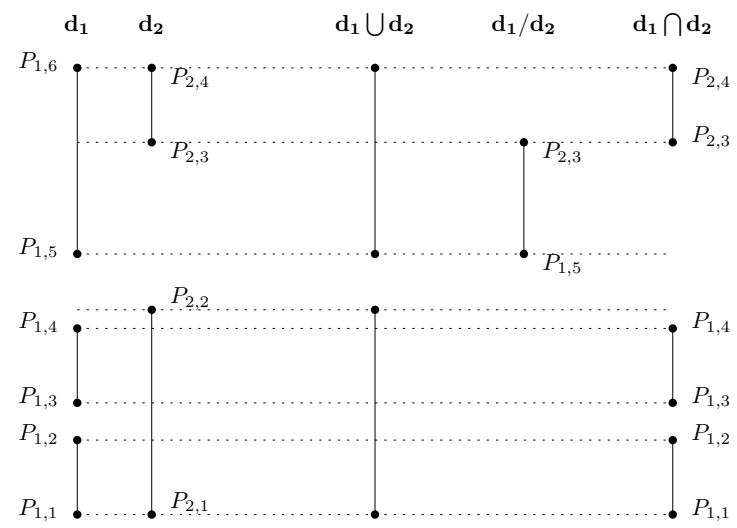

Figure 3. Boolean operations defined between dexels.

\subsection{Simulation Process}

The use of primitives, like cylinders and cuboids combined with the boolean operations allows to create simpler workpieces. After defining a milling tool and a workpiece their intersection tridexel can be calculated, which can be used to calculate the CWE. It is represented by a matrix in Figure 4. This CWE matrix can be used for calculating the forces, using various force models and edge geometries. These steps are combined into a simulation by utilizing a standard G-code input. The toolpath is subdivided into smaller segments. For every segment the cylindrical tool and swept volume are created to intersect the workpiece with. These intersections can be used to calculate the CWE and the forces.

\section{Force Models}

In the calculation of the cutting force components, it is essential to determine the relationship between the local chip thickness and the specific force. In principle, this relationship is based mainly on the material properties. The cutting force is often modelled via FEM simulations, where the material model is usually based on an elastic-viscoplastic constitutive equation extended with thermal softening effect, where the yield stress of the material is governed by the expression proposed by Johnson and Cook [7]. In spite of the advanced material description, the resultant cutting

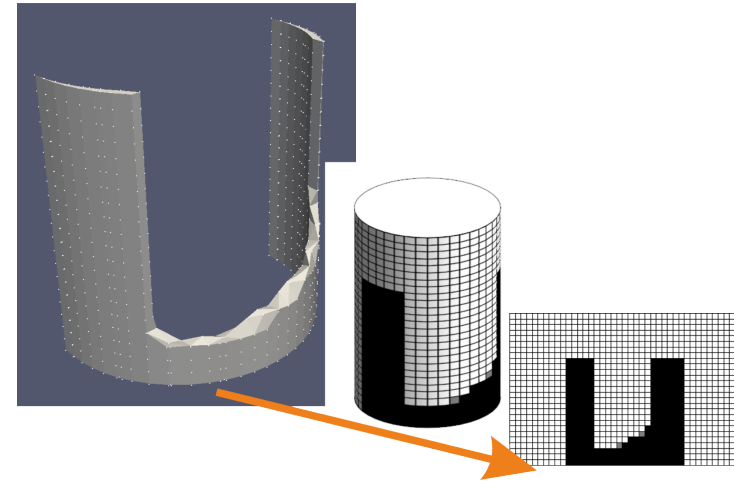

Figure 4. Main steps in generating the CWE - from left to right: intersection tridexel, CWE calculation, CWE matrix.

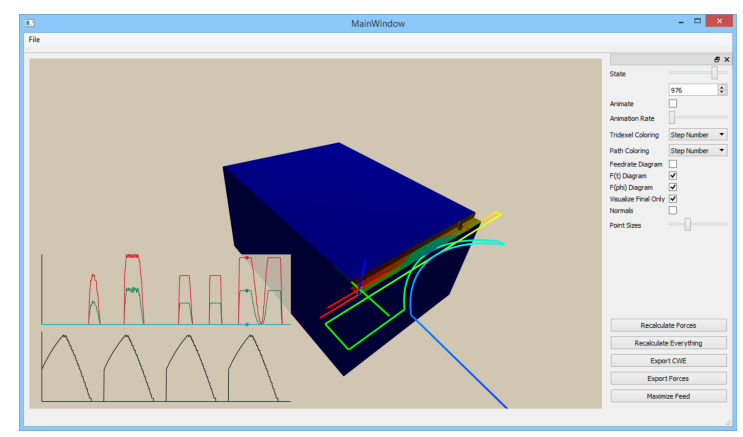

FiguRE 5. Screenshot of the software on Windows showing the simulation of the later measured case.

force has a large sensitivity on the parameters of the FEM models. In practice, the resultant cutting force is measured and then different force models are fitted, from the simplest linear (1), the shifted-linear (2), the three quarter (3) and to the third order polynomial (4) force model. The resultant coefficients are in close correlation with the material properties.

$$
\begin{aligned}
F_{\text {linear }} & =k w h, \\
F_{\text {shift }} & =k_{\text {shift }} w\left(h+h_{\text {shift }}\right), \\
F_{\text {three-quarter }} & =k_{3 / 4} w h^{3 / 4}, \\
F_{\text {polynomial }} & =w\left(Q_{0}+Q_{1} h+Q_{2} h^{2}\right),
\end{aligned}
$$

where $k$ is the cutting force coefficient, $w$ is the chip width, $h$ is the chip thickness and $Q_{i}$ are the polynomial coefficients.

\section{Experimental VAlidation}

Several cutting force measurements were carried out with constant radial and axial depth of cut with the measurement setup presented in [8] and in Figure 7]-8 Different force models were fitted for each measurement, based on the time function of the cutting force, shown in Figure 6. The coefficients shown in Table 1 have good agreement with ones presented in the literatures 2. It is found, that cutting force models with multiple parameters can lead to smaller error, however, the linear and three-quarter rule have smaller 


\begin{tabular}{|l|l|ll|l|lll|}
\hline \multicolumn{1}{|c|}{ Cases } & Linear & \multicolumn{2}{c|}{ Shifted } & \multicolumn{1}{c|}{$\mathbf{3 / 4}$} & \multicolumn{3}{c|}{ Cubic } \\
\hline & $k$ & $k_{\text {shift }}$ & $h_{\text {shift }}$ & $k_{34}$ & $Q_{0}$ & $Q_{1}$ & $Q_{2}$ \\
\hline 1 & 696.6125 & 307.4470 & 0.5448 & 976.3130 & 31.6258 & 2011.2 & -1854.6 \\
2 & 840.1928 & 503.9487 & 0.3957 & 1008.88 & 56.4329 & 902.4742 & -58.1335 \\
3 & 784.6768 & 479.4080 & 0.4464 & 871.3346 & 65.2098 & 479.9361 & 271.2453 \\
4 & 788.5676 & 785.1263 & 0.0206 & 1146 & 21.6363 & 765.2083 & 1080.9 \\
5 & 939.6342 & 664.7103 & 0.2348 & 1159 & 34.7089 & 915.6962 & 365.1631 \\
6 & 828.4690 & 624.2070 & 0.2230 & 940 & 28.8512 & 2842.1 & -2359.6 \\
7 & 869.1109 & 750.2941 & 0.0597 & 1289.6 & 14.7875 & 872.2752 & 1175,3 \\
8 & 978.0969 & 877.4342 & 0.0625 & 1231.6 & 17.8862 & 2438.7 & -1546.7 \\
9 & 841.3138 & 728.3958 & 0.1023 & 972.35 & 19.9137 & 1591.9 & -727.6420 \\
\hline Average & 840.7416 & 635.6635 & 0.2322 & 1066.12 & 32.3391 & 1424.388 & -603.671 \\
Deviation & 83.8457 & 177.8332 & 0.1906 & 144.1859 & 17.55409 & 834.6887 & 1218.447 \\
Rel. Dev. & 0.09973 & 0.2798 & 0.8208 & 0.1352 & 0.5428 & 0.5856 & -2.0184 \\
\hline
\end{tabular}

TABLE 1. Fitted parameters where the units are given as follows: $k\left[\mathrm{~N} / \mathrm{mm}^{2}\right], k_{\text {shift }}\left[\mathrm{N} / \mathrm{mm}^{2}\right], h_{\text {shift }}[\mathrm{mm}]$, $k_{3 / 4}\left[\mathrm{~N} / \mathrm{mm}^{7 / 4}\right], Q_{0}[\mathrm{~N} / \mathrm{mm}], Q_{1}\left[\mathrm{~N} / \mathrm{mm}^{2}\right], Q_{2}\left[\mathrm{~N} / \mathrm{mm}^{3}\right]$.

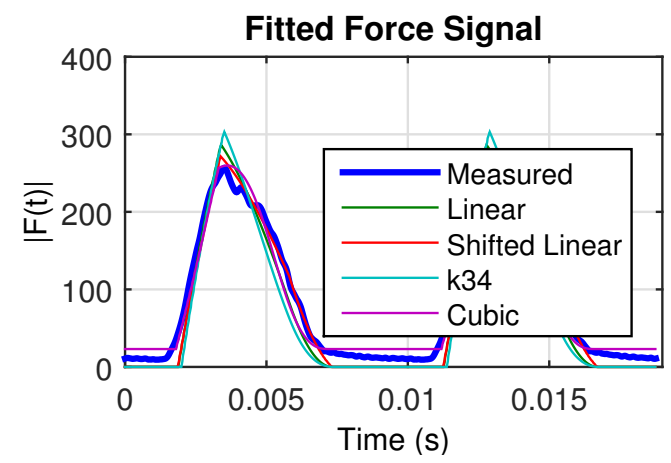

Figure 6. Comparison of various fitted force models and the actual measured force signal.

deviations between different measurement conditions. Hence, these two are good candidates for force computations at complex CWE-s.

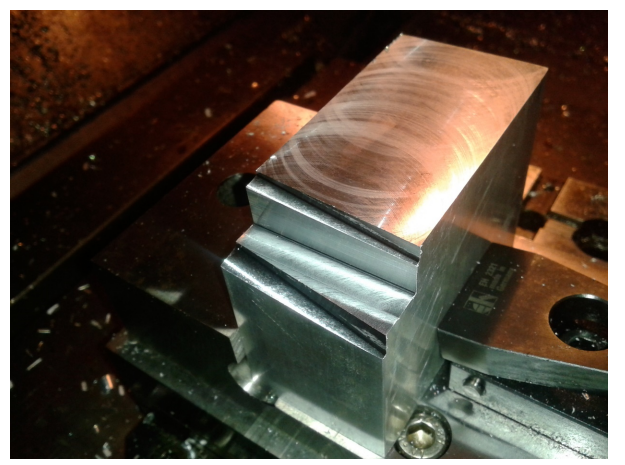

FiguRE 7. Workpiece after the measurements; on the lower side the variable axial-radial depth test can be seen.

Furthermore, a continously changing axial and radial immersion toolpath was used as shown in Figure7. The CWE was computed based on the multi-dexel solid modelling and the resultant cutting force is computed for the linear force model. Figure 9 presents the measured and the calculated forces, respectively.

Finally, an experiment was conducted with a more

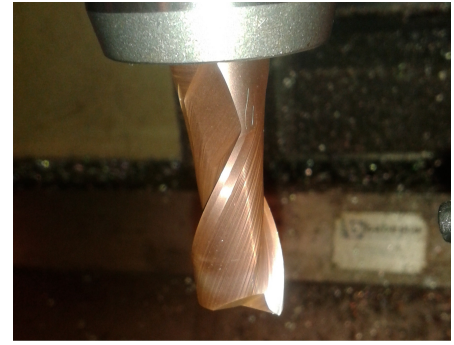

Figure 8. The tool used for the force measurements.

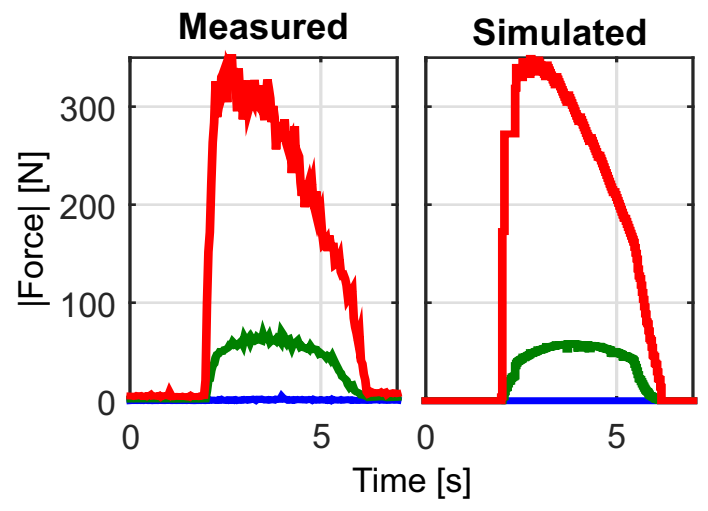

Figure 9. Comparison of the measured and the simulated force signal in the case of continously varying axial and radial immersions. - - Maximum force, $\square$ - average force, $\mathbf{\square}$ - minimum force.

complex toolpath, where a circular arc was investigated (see Figure 5), on which we tested the force signal by up- and downmilling procedures as well.

Based on the very good agreement shown in Figure 9 11 it can be stated, that the simplest force functions (linear, three-quarter) can be used to compute the resultant cutting force precisely in milling, if the CWE is determined with high accuracy.

During testing of the more complex toolpaths (Figure 5, Figure 10 11) we were faced with the fact that our calculated force signal was qualitatively the same as the measured, but the exact timings were not 


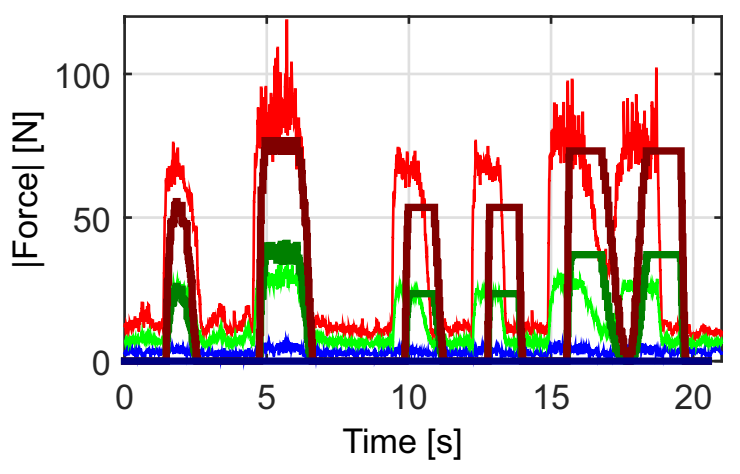

Figure 10. Comparison of the measured and the simulated force signal in the case of down- and upmilling. Measured forces: $\mathbf{\square}$ - maximum, $\mathbf{\square}$ - average, $\mathbf{\square}$ - minimum; Calculated forces: $\mathbf{\square}$ - maximum, $\mathbf{\square}$ - average, 口 - minimum.

correct. After investigating the effect it was concluded that this comes from the fact that in our calculations it was assumed that the milling machine can follow the prescribed speeds perfectly and did not take into account the control. In reality, this assumption is false, since these machines have their own control module, which could smooth out the sharp direction changes due to the limited jerk and accelerations. The milling machine, on which the measurements where carried out, is not an industrial machine, and in case of sharp direction changes the machine slows down and when it runs off of the material it speeds up more considerably. Because the goal of the measurement was to validate only the forces the time signal was corrected by inserting small delays where needed, with the sum of 0.675 seconds.

\section{Conclusions and Future RESEARCH}

From the experiments it can be concluded that the calculation of the forces is sufficiently good and the results are promising. There is a good agreement between the calculated and the measured data despite not taking the control dynamics of the milling machine into account. The computed results can be used for feed optimization, also the cutting process of a workpiece can be investigated deeper to avoid the chatter and stability losses, so it can also help manufacture items of higher quality faster. In the future the expansion of the software with the above mentioned functions will be pursued.

\section{ACKNowledGements}

This paper was supported by the Hungarian Scientific Research Fund - OTKA PD-112983 and the Janos Bolyai Research Scholarship of the Hungarian Academy of Sciences.

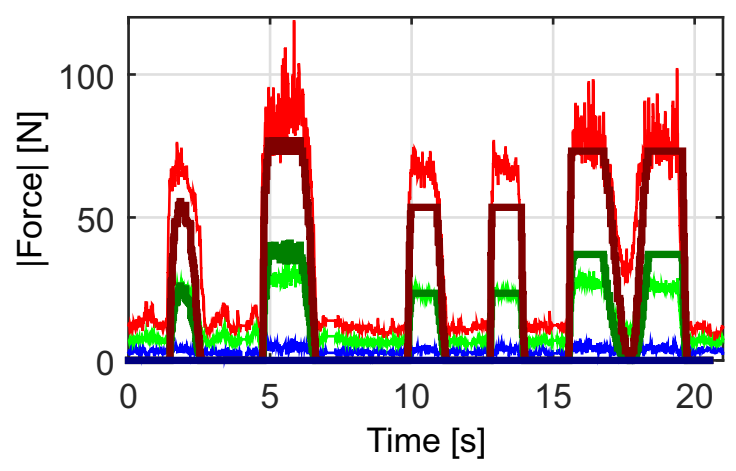

Figure 11. Comparison of the corrected measured and the simulated force signal in the case of down- and upmilling. Measured forces: $\mathbf{0}$ - maximum, $\mathbf{\square}$ - average, - minimum; Calculated forces: $\mathbf{\square}$ - maximum, - - average, $\mathbf{\square}$ - minimum.

\section{REFERENCES}

[1] Y. Altintas, P. Kersting, D. Biermann, et al. Virtual process systems for part machining operations. CIRP Annals - Manufacturing Technology 63(2):585-605, 2014. DOI:10.1016/j.cirp.2014.05.007

[2] D. Bachrathy. Cutting dynamics and surface quality. Ph.d thesis, Budapest University of Technology and Economics, 2013.

[3] Y. Ren, W. Zhu, Y.-S. Lee. Feature Conservation and Conversion of Tri-dexel Volumetric Models to Polyhedral Surface Models for Product Prototyping. Computer-Aided Design and Applications 5(6):932-941, 2008. DOI:10.3722/cadaps.2008.932-941

[4] X. Qian, J. Villarrubia. General three-dimensional image simulation and surface reconstruction in scanning probe microscopy using a dexel representation.

Ultramicroscopy 108(1):29-42, 2007. DOI:10.1016/j.ultramic.2007.02.031.

[5] B. Tukora. Material Removal Simulation and Cutting Force Prediction of Multi-Axis Machining Processes on General-Purpose Graphics Processing Units. Ph.d thesis, Budapest University of Technology and Economics, 2012.

[6] R. Anderson. Detecting and eliminating collisions in NC machining. Computer-Aided Design 10(4):231-237, 1978. DOI:10.1016/0010-4485(78)90058-1.

[7] G. R. Johnson, W. H. Cook. Fracture characteristics of three metals subjected to various strains, strain rates, temperatures and pressures. Engineering Fracture Mechanics 21(1):31-48, 1985. DOI:10.1016/0013-7944(85)90052-9

[8] I. Biró, M. Czampa, T. Szalay. Experimental Model for the Main Cutting Force in Face Milling of a High Strength Structural Steel. Periodica Polytechnica Mechanical Engineering 59(1):16-22, 2014. DOI:10.3311/PPme.7516. 